\title{
Effect of bilayer charge on lipoprotein lipid exchange
}

\author{
Kathryn Louise Browning ${ }^{\mathrm{a}, \mathrm{b}^{*}}$, Tania Kjellerup Lind ${ }^{\mathrm{c}}$, Selma Maric ${ }^{\mathrm{c}}$, Robert David Barker ${ }^{\mathrm{d}, 1}$, Marité \\ Cárdenas ${ }^{\mathrm{c}^{*}}$ and Martin Malmsten ${ }^{\mathrm{a}, \mathrm{b}}$ \\ ${ }^{a}$ Department of Pharmacy, Uppsala University, Uppsala, Sweden. \\ ${ }^{\mathrm{b}}$ Department of Pharmacy, University of Copenhagen, Copenhagen, Denmark. \\ ${ }^{\mathrm{c}}$ Department of Biomedical Sciences and Biofilms - Research Centre for Biointerfaces, Malmö \\ University, Malmö, Sweden \\ ${ }^{\mathrm{d}}$ Institut Laue-Langevin, Grenoble, France
}

Present address

${ }^{1}$ School of Physical Sciences, University of Kent, Canterbury, UK

Corresponding author contact details:

Kathryn Browning

Department of Pharmacy,

University of Copenhagen,

Universitetsparken 2,

Copenhagen 2100,

Denmark

kathryn.browning@sund.ku.dk

\section{Marité Cárdenas}

Department of Biomedical Sciences and Biofilms,

Malmö University,

Malmö 20506,

Sweden

marite.cardenas@mah.se 


\section{Abstract}

Lipoproteins play a key role in the onset and development of atherosclerosis, the formation of lipid plaques at blood vessel walls. The plaque formation, as well as subsequent calcification, involves not only endothelial cells but also connective tissue, and is closely related to a wide range of cardiovascular syndromes, that together constitute the number one cause of death in the Western World. High (HDL) and low (LDL) density lipoproteins are of particular interest in relation to atherosclerosis, due to their protective and harmful effects, respectively. In an effort to elucidate the molecular mechanisms underlying this, and to identify factors determining lipid deposition and exchange at lipid membranes, we here employ neutron reflection (NR) and quartz crystal microbalance with dissipation (QCM-D) to study the effect of membrane charge on lipoprotein deposition and lipid exchange. Dimyristoylphosphatidylcholine (DMPC) bilayers containing varying amounts of negatively charged dimyristoylphosphatidylserine (DMPS) were used to vary membrane charge. It was found that the amount of hydrogenous material deposited from either HDL or LDL to the bilayer depends only weakly on membrane charge density. In contrast, increasing membrane charge resulted in an increase in the amount of lipids removed from the supported lipid bilayer, an effect particularly pronounced for LDL. The latter effects are in line with previously reported observations on atherosclerotic plaque prone regions of long-term hyperlipidaemia and type 2 diabetic patients, and may also provide some molecular clues into the relation between oxidative stress and atherosclerosis.

\section{Keywords}

Atherosclerosis, HDL, LDL, Lipid exchange, Lipoprotein, Neutron Reflection 


\section{Introduction}

Arteriosclerosis and its clinical complications have been identified as the largest cause of mortality this century.[1] Development of atherosclerotic plaques and lesions is thought to be initiated by transfer of cholesterol from low density lipoprotein (LDL), oxidized LDL, lipoprotein(a), and some other lipoproteins to the blood vessel wall. This induces foam cell formation and eventually calcification. The latter results in a dramatic stiffening of the blood vessels and an effective reduced diameter, increasing the risk of blockage and/or rupture of the vessel, leading to ischemic heart attack and stroke.[2, 3]

Lipids are transported to and from peripheral cells by lipoproteins; nanoscopic packages containing primarily cholesterol esters and triglycerides, coated by a monolayer of lipids and apolipoproteins.[4] Lipoproteins are categorised by density and size, and different classes are thought to play important roles in the development of, and protection from, atherosclerosis.[4] Among these, LDL and high density lipoprotein (HDL) are of particular interest to atherosclerosis. LDL particles are larger than HDL and contain higher levels of cholesterol esters. High levels of LDL in the blood are linked to increased risk of atherosclerosis,[5] whereas high HDL levels are associated with reduced atherosclerotic risk.[6] Consequently, LDL (also known as the 'bad cholesterol') levels are currently used as an atherosclerotic risk indicator, although the ratios of LDL to either HDL ('good cholesterol') or total cholesterol content are becoming more prevalent due to HDL's role in reverse cholesterol transport to the liver and the atheroprotective effects of HDL.[4] 
Surface sensitive techniques can be employed to investigate the interaction of lipoproteins with (model) cell membranes by supporting them on solid surfaces. We previously used neutron reflection [7] and quartz crystal microbalance with dissipation (QCM-D)[8] to study the interaction of LDL and HDL with synthetic supported lipid bilayers (SLBs).[9] Using deuterated as well as non-deuterated SLBs formed of 90 mol\% dimyristoylphosphatidylcholine (DMPC) and 10 mol\% dimyristoylphosphatidylserine (DMPS), which has a negative charge close to that expected for endothelial cell membranes.[10, 11] Both HDL and LDL were shown to remove lipids from the SLB and also to deposit hydrogenous material into the bilayer.[9] However, lipid deposition from lipoprotein to SLB was higher for LDL than for HDL, whereas HDL displayed a considerably higher extent of lipid removal from the SLB. These results correlate well to the clinically observed 'bad' and 'good' effects of LDL and HDL on atherosclerosis development, respectively.

In the present work, we aim to extend our studies into the molecular mechanisms underlying atherosclerosis by investigating the effect of bilayer charge on the adsorption of lipoproteins and lipid dynamics between the particles and the bilayer, as the literature contains seemingly conflicting data on this. For example, lipoproteins bind extensively to negatively charged surfaces, forming the basis for removal of lipoproteins from bloodstream circulation through apheresis using various polyanionic macromolecules (e.g., heparin, dextran sulfate, and sulfated poly(vinyl alcohol)).[12-17] Therefore, one would expect lipoprotein binding to increase with increasing negative membrane charge. However, long-term hyperlipidaemia and type 2 diabetic patients display a reduction of anionic groups in endothelial cells in atherosclerotic lesion-prone regions, which could suggest greater interaction of LDL with lower charged membranes.[18-20] Consequently, there is a need to better understand the role 
of membrane charge on the extent of lipoprotein binding and their dynamics at model cellular membranes.

In addressing the effects of membrane charge density, DMPC/DMPS bilayers were prepared at different molar ratios, and their effect on LDL binding and lipid dynamics was monitored by neutron reflectometry and QCM-D. Morphological changes and phase separation of PCand PS-lipids perpendicular to the bilayer plane have previously been reported for multilamellar vesicles above $30 \mathrm{~mol} \%$ PS. Therefore, concentrations of PS up to $25 \mathrm{~mol} \%$ were used to ensure the formation of homogenous flat bilayers at silicon surfaces.[21] Through this, increasing membrane charge was demonstrated to result in an increase in the amount of lipids removed from the SLB, an effect particularly pronounced for LDL, whilst the amount of hydrogenous material deposited from the lipoprotein to the SLB was found to be largely independent of membrane negative charge.

\section{Experimental}

\section{Materials}

Tail deuterated DMPC (1,2-dimyristoyl-d54-sn-glycero-3-phosphocholine) and DMPS (1,2dimyristoyl-sn-glycero-3-phospho-L-serine), as well as their non-deuterated equivalents of $>99 \%$ purity, were obtained from Avanti Polar Lipids (Alabaster, USA). Ultrapure water (18.2 $\mathrm{M} \Omega \mathrm{cm}^{-1}$, Millipore) and $\mathrm{D}_{2} \mathrm{O}$ (99.9 \% deuterated, Sigma Aldrich) were used throughout. Tris buffer was prepared by dissolving a buffer tablet (Sigma Aldrich) to obtain buffer solutions of $50 \mathrm{mmol} \mathrm{L}^{-1}$ Tris, $150 \mathrm{mmol} \mathrm{L}^{-1} \mathrm{NaCl}$, $\mathrm{pH} 7.6$, pre-adjusted for $\mathrm{pH}$ in either $\mathrm{H}_{2} \mathrm{O}$ or $\mathrm{D}_{2} \mathrm{O}$. Chloroform anhydrous $\geq 99 \%$ and calcium chloride dihydrate $(\geq 99 \%)$ were obtained from Sigma Aldrich. All other chemicals used were of analytical grade. 


\section{Preparation of lipoproteins}

Lipoproteins were prepared by sequential ultracentrifugation (densities of 1.065 and $1.019 \mathrm{~g}$ $\mathrm{mL}^{-1}$ for HDL and LDL, respectively) of plasma pooled from three healthy males. The samples were stored in 50\% sucrose, $150 \mathrm{mmol} \mathrm{L}^{-1} \mathrm{NaCl}, 24 \mathrm{mmol} \mathrm{L}^{-1}$ EDTA, $\mathrm{pH} 7.4$, at $80^{\circ} \mathrm{C}$. Before use, buffer was exchanged to $50 \mathrm{mmol} \mathrm{L}^{-1}$ Tris, $150 \mathrm{mmol} \mathrm{L} \mathrm{L}^{-1} \mathrm{NaCl}, \mathrm{pH} 7.4$, using a Sephadex G25 PD-10 desalting column (GE Healthcare, Uppsala, Sweden) and further purified by size exclusion chromatography (Superose 6 Increase 10/300 GL column, GE Healthcare, Uppsala, Sweden) at $25^{\circ} \mathrm{C}$. Each fraction was then stored away from light, at $4^{\circ} \mathrm{C}$, under an inert atmosphere. Prior to use the protein concentration was determined by Bradford analysis [22] and the solutions diluted to either $0.132 \mathrm{mg} \mathrm{mL}^{-1}$ (HDL) or $0.1 \mathrm{mg}$ $\mathrm{mL}^{-1}$ (LDL), concentrations chosen to maintain a constant particle concentration of HDL and LDL, calculated from the protein content per lipoprotein particle.[23] Preliminary experiments showed pronounced changes to the bilayer, induced by the lipoproteins (either through exchange or destruction), so this concentration was chosen to allow studies of exchange and deposition effects without risking complete supported bilayer destabilisation.

\section{Preparation of lipid bilayers}

Phospholipid bilayers were deposited on the native oxide of polished silicon blocks (neutron reflection; NR) or silicon dioxide surfaces (QCM-D) by vesicle fusion. In short, lipid films were prepared by dissolving DMPC in chloroform and DMPS in a 2:1 chloroform:methanol solution. Non-deuterated lipids were used for QCM-D measurements and tail-deuterated lipids for NR. The lipids were then mixed in appropriate molar ratios and dried to the walls of clean glass vials by evaporation with nitrogen. The films were further dried under vacuum for 24 hours at room temperature. Before use the lipid films, consisting of 100 mol\% DMPC:0 
mol\% DMPS), 90 mol\% DMPC:10 mol\% DMPS, or 75 mol\% DMPC:25 mol\% DMPS , were hydrated for 1 hour at $40{ }^{\circ} \mathrm{C}$ in order to be above the phase transition temperature for both dDMPC $\left(19^{\circ} \mathrm{C}\right)$ and dDMPS $\left(31^{\circ} \mathrm{C}\right)$.[24] Hydrated films were then sonicated before injection using a tip sonicator (Hielscher, Germany) intermittently for 5 minutes until clarity whilst ensuring the temperature did not rise above $50^{\circ} \mathrm{C}$. Silicon surfaces were first washed with $2 \mathrm{mmol} \mathrm{L}^{-1} \mathrm{CaCl}_{2}$ solution before a 1:1 mixture of vesicles and $4 \mathrm{mmol} \mathrm{L}^{-1} \mathrm{CaCl}_{2}$ was pumped into the cell to screen the charge and bridge between the negative silicon oxide surface and lipids. All bilayers were allowed to incubate for 20 minutes before rinsing with 5 $\mathrm{mL}$ of $2 \mathrm{mmol} \mathrm{L}^{-1} \mathrm{CaCl}_{2}$ solution, $5 \mathrm{~mL} \mathrm{H}_{2} \mathrm{O}$ and $15 \mathrm{~mL}$ Tris buffer before measurement.

\section{Quartz Crystal Microbalance with Dissipation}

Experiments were performed on a Q-Sense E4 quartz crystal microbalance (Q-Sense, Göteborg, Sweden). All experiments were measured at $37{ }^{\circ} \mathrm{C}$ in duplicate. Tubing, cells, and o-rings were cleaned first in 2\% Hellmanex solution (Sigma Aldrich), rinsed in ultra-pure water and ethanol (99.9\%, Sigma Aldrich) before drying under nitrogen. Silicon oxide sensors were cleaned in the same way before UV-Ozone treatment for 10 minutes (BioForce Procleaner, Bioforce Nanosciences, Salt Lake City, USA), resulting in highly hydrophilic surfaces fully wettable by water (contact angles of less than $10^{\circ}$ ). Resonance frequencies were obtained in ultra-pure water and bilayers formed as described above using a flow rate of $100 \mu \mathrm{L} \min ^{-1}$ until stable signals, characteristic for complete bilayers $\left(\Delta \mathrm{F} \approx-25 \mathrm{~s}^{-1}, \Delta \mathrm{D} \approx 0\right.$ a.u.), were established.[25] The bilayers were then washed in Tris buffer at $100 \mu \mathrm{L} \mathrm{min}^{-1}$ for 20 minutes. Subsequently, $1 \mathrm{~mL}$ of either HDL $\left(0.132 \mathrm{mg} \mathrm{mL}^{-1}\right.$, based on protein content) or LDL ( $0.1 \mathrm{mg} \mathrm{mL}^{-1}$ based on protein content) was pumped into the measurement cell at 100 $\mu \mathrm{L} \min ^{-1}$, whereafter the pump was stopped and the lipoproteins allowed to incubate for 12 
hours at $37^{\circ} \mathrm{C}$. The sample was then washed for 30 minutes at $100 \mu \mathrm{L} \mathrm{min}{ }^{-1}$ with Tris buffer and no change was detected upon rinsing.

\section{Neutron Reflection}

Neutron reflectivity measurements were carried out at the horizontal reflectometer FIGARO at Institut Laue-Langevin, France.[26, 27] The reflectometer was set in the reflection up mode such that any particulates or aggregated lipoproteins settled at the bottom of the cell away from the reflection interface. Polished silicon $\{111\}$ blocks $(80$ x 50 x 15 mm, Sil'tronix, France) were cleaned in a dilute piranha solution (5 parts $\mathrm{H}_{2} \mathrm{O}, 4$ parts $\mathrm{H}_{2} \mathrm{SO}_{4}$ and 1 part $\mathrm{H}_{2} \mathrm{O}_{2}$ ) at between 80 and $85^{\circ} \mathrm{C}$ for 10 minutes before extensive rinsing in ultrapure water. The crystal surfaces were dried under a nitrogen stream and further cleaned using a UV ozone cleaner for 15 minutes to remove organic contamination and improve the hydrophilicity of the surface. The block was again rinsed with ultrapure water and immediately clamped against a polyether ether ketone (PEEK) trough with a Viton O-ring (both cleaned in 2\% Hellmanex solution and ultrapure water with sonication), which was full to meniscus with ultra-pure water. The solution was then exchanged to $\mathrm{D}_{2} \mathrm{O}$ by syringe through HPLC connections to improve the contrast for alignment and to check the seal of the reflectivity cell against the silicon block. The temperature for all measurements was set to 37 ${ }^{\circ} \mathrm{C}$ and maintained by circulating water bath. Measurements were taken in the time-of-flight mode at two incident angles, 0.8 and $3.2^{\circ}$, to cover the $\mathrm{Q}$ range of interest $\left(0.01-0.25 \AA^{-1}\right)$. The footprint of the neutron beam on the reflection interface was set by collimation slits and kept constant between the two incident angles. An experimental resolution of $\delta \lambda / \lambda=7 \%$ was used to provide a good compromise between spatial resolution and count times. Silicon blocks were first characterised in $\mathrm{D}_{2} \mathrm{O}$ and $\mathrm{H}_{2} \mathrm{O}$ to obtain the roughness and thickness of the 
oxide layer and to ensure cleanliness of the surface. Lipid bilayers were then formed on the silicon surface as described above. Each bilayer was then characterised in three contrasts, Tris/ $\mathrm{H}_{2} \mathrm{O}$ (hTris), Tris/ $\mathrm{D}_{2} \mathrm{O}$ (dTris), and Tris solution with the same scattering length density as silicon $\left(\mathrm{CMTris}=2.07 \times 10^{-6} \AA^{-2}\right)$, using a HPLC pump to exchange the solution in the cell.

Lipoproteins (HDL $\left(0.132 \mathrm{mg} \mathrm{mL}^{-1}\right.$, based on protein content) or LDL $\left(0.1 \mathrm{mg} \mathrm{mL}^{-1}\right.$ based on protein content) in hTris were introduced to the cell by syringe pump at $1 \mathrm{~mL} \mathrm{~min}{ }^{-1}$ and incubated for 8 hours with kinetic measurements every minute (single angle, $0.8^{\circ}$ ) for the first hour and full characterisation (both angles) every two hours during incubation. After the final measurement, the lipoproteins in solution were immediately rinsed away with $20 \mathrm{~mL}$ hTris and again characterised in three contrasts. Lipid removal was found to be essentially halted by washing the bilayer and removal of lipoproteins in solution as shown by the change after washing at 10 hours (Figure 5). After characterisation in three contrasts (6 hours), a final lipid removal check in hTris was performed that showed $0-4 \%$ lipid removal during total characterisation. Data was fitted using the MOTOFIT program which uses the Abeles formulism to calculate the expected reflectivity profile.[28] A constant sample background was first fitted, then fixed, for each contrast. For dTris and CMTris contrasts, the background was set to $6 \times 10^{-7}$, a higher background of $1 \times 10^{-6}$ was needed to model hTris contrasts due to the higher incoherent scattering of hydrogen. After lipoprotein introduction, the background was found to be higher $\left(8 \times 10^{-7}\right.$ for dTris and CMTris and $1.5 \times 10^{-6}$ for hTris). When comparing with data in the supported information from INTER (ISIS, Rutherford Appleton Laboratory, UK) it should be noted that the sample background at INTER is not subtracted and is higher $\left(5.5-8 \times 10^{6}\right)$.

\section{Results}




\section{Effect of bilayer charge on lipoprotein adsorption}

The lipoprotein adsorption to SLBs with increasing levels of negative charge $(0 \mathrm{~mol} \%$ DMPS, 10 mol\% DMPS or 25 mol\% DMPS) was first investigated using QCM-D (Figure 1). Following a lag time upon exposure to the SLBs, both LDL and HDL induced a decrease in frequency and an increase in dissipation, indicating an increased sensed wet mass and an increased 'softness' of the interfacial region due to lipoprotein adsorption for all membrane charge densities. Not only the SLB charge but also the lipoprotein type determined the extent of change in the QCM-D signals. For LDL, the absolute change in frequency and dissipation, as well as the rate of the change in both signals, increased with PS content in the SLBs. In contrast, the lag time prior to the change in the QCM-D signals decreased with charge (PS content), being essentially instantaneous for the 25 mol\% DMPS SLB. Such change in QCMD signals could be interpreted as an increase in the rate of LDL adsorption with increasing negative charge of the SLBs. Interestingly, the dissipation signal at $25 \mathrm{~mol} \%$ DMPS was suppressed as compared to $10 \mathrm{~mol} \%$ DMPS or $0 \mathrm{~mol} \%$ DMPS, most likely due to differences in the lipoprotein adsorbed structure (discussed further below).[29] The lower decrease in frequency and dissipation observed for HDL suggests lower adsorption of this lipoprotein as compared to LDL for the SLBs investigated. This result was largely independent of bilayer charge. Furthermore, a low dissipation signal was observed, particularly so for $25 \mathrm{~mol} \%$ DMPS, indicating that the rigidity of the surface (and the supported bilayer) was not affected by HDL binding and that HDL possibly binds more tightly to the SLB interface.

Figure 1: QCM-D data showing the change in frequency (bottom, blue) and dissipation (top, red) for bilayers incubated with either $0.1 \mathrm{mg} \mathrm{mL}^{-1}$ of LDL (left) or $0.132 \mathrm{mg} \mathrm{mL}^{-1}$ of HDL (right). Three bilayers are shown with increasing negative charge: dark colours refer to DMPC bilayers, mid colours to 10 mol\% DMPS: 90 mol\% DMPC bilayers (data taken from 
Browning et.al.)[9] and pale colours to $25 \mathrm{~mol} \%$ DMPS: 75 mol\% DMPC bilayers. Three overtones are plotted for clarity:

$$
7^{\text {th }} \text { (solid line), } 9^{\text {th }} \text { (dashed line) and } 11^{\text {th }} \text { (dotted line). }
$$

LDL
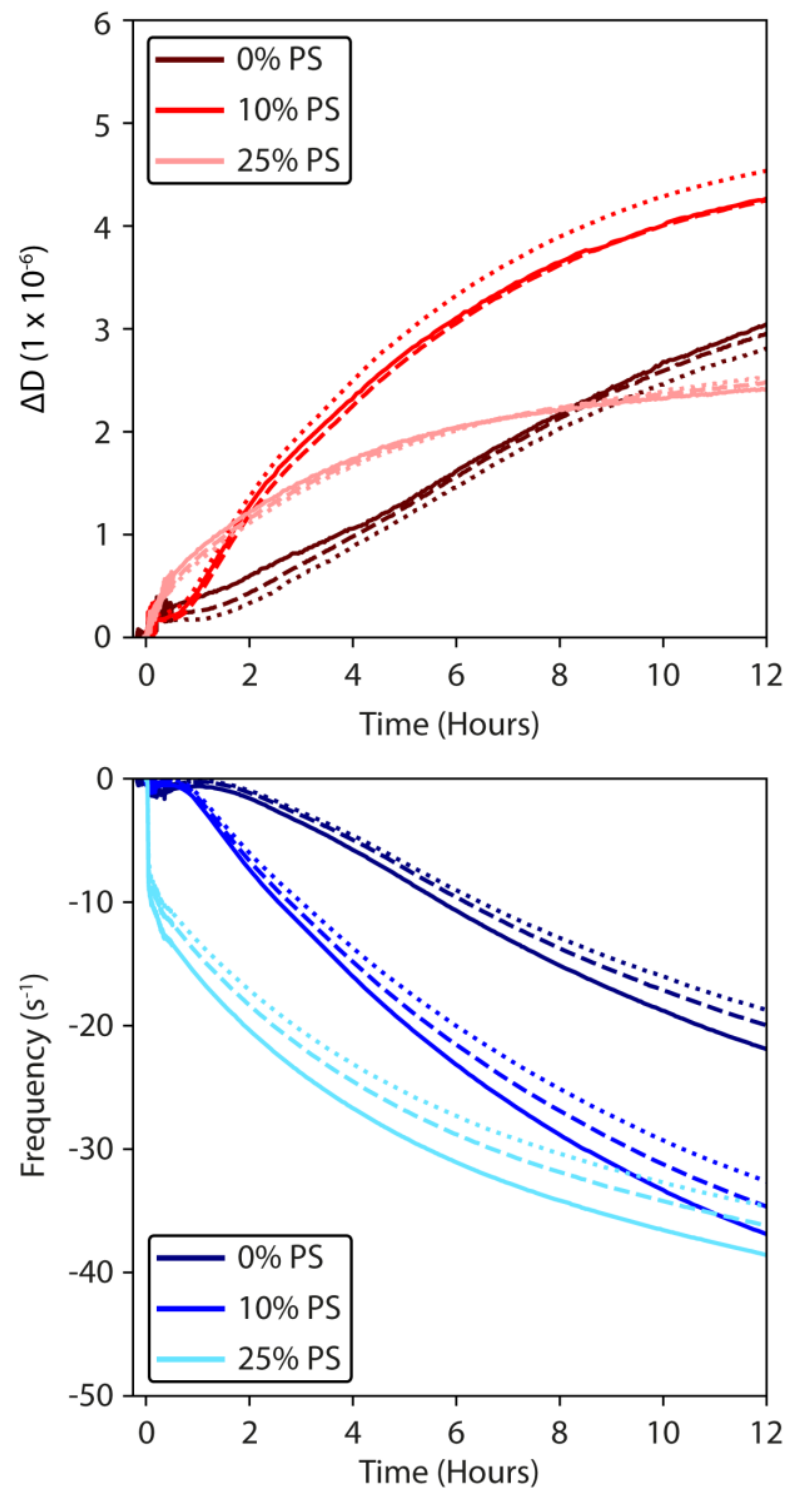

$\mathrm{HDL}$
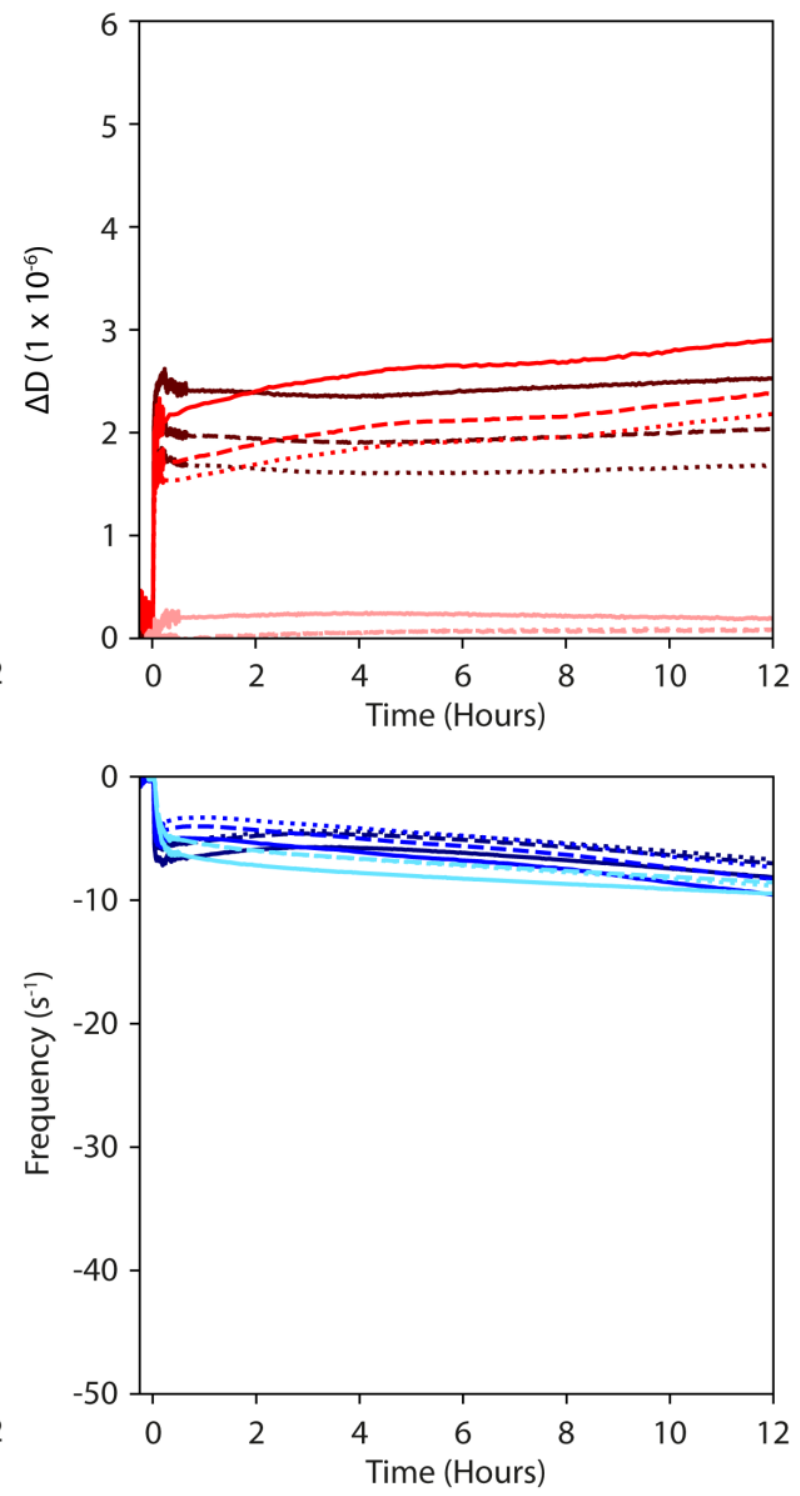

\section{Effect of bilayer charge on lipid exchange}

In order to obtain more detailed information on the structural effects of HDL and LDL on the

SLBs, as well as lipid exchange processes, we next performed neutron reflectometry experiments, examining tail-deuterated SLBs before and after contact with hydrogenous lipoproteins in various $\mathrm{H}_{2} \mathrm{O} / \mathrm{D}_{2} \mathrm{O}$ contrasts. In doing so, we compared our previously 
published results on 10 mol\% DMPS: 90 mol\% DMPC bilayers [9] with those on DMPC SLBs containing either 0 or 25 mol\% DMPS. Scattering length densities of the materials used are summarised in SI Table 1 and all fitted parameters for the SLBs before and after lipoprotein incubations (SI Table 2) are given as Supplementary Information.

\section{DMPC/DMPS 100/0}

Figure 2 shows the change in reflectivity from a 0 mol\% DMPS bilayer upon incubation of both LDL (top) and HDL (bottom) with the original bilayer data underlaid in lighter colours. Three contrast datasets for the 0 mol\% DMPS bilayers, prior to lipoprotein addition, were fitted simultaneously along with the bare surface and could be well fitted, agreeing with literature values for the head and tail region thickness and roughness.[30] The SLD of the tail region was found to be slightly lower than previously reported in literature, an effect due to incomplete deuteration of the tail region, as discussed previously.[9] Nevertheless, the bilayer coverage was found to be high, with no detectable water in the tail region and an area per molecule of $60 \pm 1 \AA^{2}$. Fitted parameters, SI Table 1, and calculated SLD profiles, SI Figure 1, are shown as Supplementary Information.

After lipoprotein incubation, the reflectivity of the bilayers decreased, indicating changes to both bilayer structure and composition. Measurements performed in hTris contrast (blue) are sensitive to the amount of deuterated material remaining in the bilayer. A drop in the reflectivity thus indicates lipid removal but does not differentiate between replacement with hydrogenous lipids through an exchange mechanism, or filling the resulting voids with water (removal). In order to decouple the exchange and removal processes, the data obtained in dTris is much more useful. For the latter, a change in the SLD of the tail region indicates 
insertion of hydrogenous material, i.e., exchange rather than removal. The difference between the two values then gives the amount of deuterated lipids removed from the bilayer and not replaced.

Figure 2: Experimental (markers) and fitted (lines) neutron reflectivity data showing the effect of lipoprotein addition $(0.1$ $\mathrm{mg} \mathrm{mL} \mathrm{L}^{-1} \mathrm{LDL}$, top and $0.132 \mathrm{mg} \mathrm{mL}^{-1} \mathrm{HDL}$, bottom) to $0 \mathrm{~mol} \%$ DMPS SLBs in three contrasts, dTris (green), CMTris (orange) and hTris (blue). Data and fits from the original bilayers are underlaid in pale colours. For clarity, data for CMTris and dTris are offset by 10 and 1000, respectively.

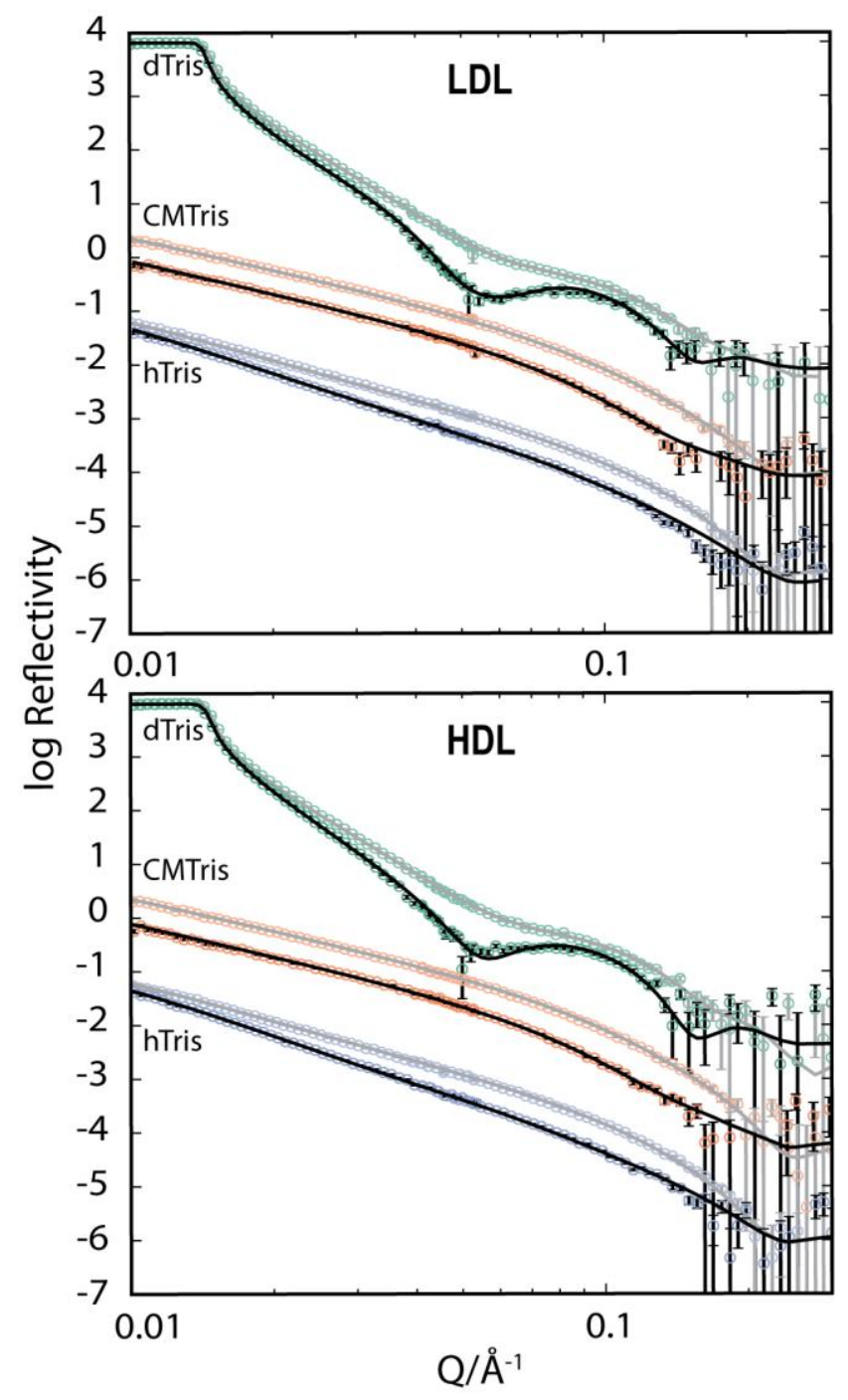


Strikingly similar changes in reflectivity were observed for LDL and HDL on 0 mol\% DMPS SLBs (Figure 2). The major difference was seen in the hTris contrast, where the reduction in reflectivity was slightly greater for HDL compared to LDL. This suggests greater removal of deuterated lipids from the SLB for HDL. When three contrasts for each bilayer were fitted simultaneously, the modelled results are consequently similar with a fitted tail SLD of $4.51 \mathrm{x}$ $10^{-1} \AA^{-2}$ for LDL and $4.47 \times 10^{-1} \AA^{-2}$ for HDL (corresponding to 30 and $31 \% \pm 1 \%$ exchange, respectively). This result suggests that, for zwitterionic bilayers, the deposition of hydrogenous material (i.e., from the hydrogenous lipoproteins to the deuterated bilayer) was the same for both lipoproteins. The main difference between the lipoproteins is that removal and deposition within the tail region of the bilayer are equal for LDL, while removal is substantially higher ( $9 \%$ more) than deposition for HDL. The tail region for LDL can be fitted to have the same SLD in all contrasts, which, assuming a similar density and hydrogenous SLD, means all removed deuterated molecules are replaced within the bilayer with no detectable water. In other words, only lipid exchange takes place for LDL.

For both LDL and HDL, the outer head group region of the bilayer thickened from 9 to $17 \AA$ on lipoprotein exposure. However, the corresponding increase in hydration of the layers was not sufficient to account for the head group thickness change. The SLB of the head group should not be significantly affected by hydrogenous phospholipid exchange from the lipoproteins since the lipids used in the SLB have a non-deuterated head group. Therefore, such thickening suggests that material from the lipoprotein resides in this layer, either in the form of exchanged material with a different SLD in the head group region or directly as adsorbed lipoproteins. In relation to the latter, it was necessary to include a diffuse rough layer in order to fit the data, especially at low $\mathrm{Q}$ where changes in reflectivity from thick layers are most clearly seen. The coverage of this layer was low, however, amounting to $3 \pm$ 
$1 \%$ for both HDL and LDL. Hence, only a few lipoprotein particles remain in contact with the bilayer after washing.

\section{DMPC/DMPS 90/10}

In our previous study[9] of 10 mol\% DMPS bilayers, we found that the amount of deposited hydrogenous material was roughly similar but slightly higher for LDL (31\%) compared to HDL (26\%). Thus, the addition of $10 \mathrm{~mol} \%$ DMPS molecules to the bilayer not only increases lipoprotein adsorption (Figure 1), but also the amount of lipids removed from the SLB for both HDL and LDL. Quantitatively, the amount of lipids removed was found to be $44 \%$ for LDL and $52 \%$ for HDL, an increase of $14 \%$ and $13 \%$, respectively, compared to 0 mol\% DMPS.

\section{DMPC/DMPS 75/25}

Representing even higher negative charge density, 75 mol\% DMPC: 25 mol\% DMPS SLBs were next investigated. The paler underlaid data and fitted lines in Figure 3 show the reflectivity data from $25 \mathrm{~mol} \%$ DMPS bilayers before lipoprotein exposure, characterised in three contrasts and the data fitted simultaneously with the bare surface. The best fit to the data was found if the charged head group layers were slightly thinner and less hydrated than for the pure DMPC bilayers, in agreement with previous findings [9, 31]. MD simulations showed a reduction in bound water in the head group region of POPC and POPS bilayers, as well as head group re-orientation, in the presence of calcium ions.[32] Calcium ions are present in our system during deposition to screen the surface charge and bridge the negative 
phospholipids to the negative silicon surface and may induce this head group thinning. A thin layer of water between the silica and the SLB of the same thickness as the surface roughness was also needed to improve the fit. Similarly to $0 \mathrm{~mol} \%$ DMPS, the SLB surface coverage was found to be high $(99 \% \pm 1 \%)$, with an area per molecule $=58 \pm 1 \AA^{2}$, in agreement with previous findings.[33] Fitted parameters (SI Table 1), and calculated SLD profiles (SI Figure 1) are shown as Supplementary Information.

Figure 3: Experimental (markers) and fitted (lines) neutron reflectivity data showing the effect of lipoprotein addition (0.1 $\mathrm{mg} \mathrm{mL}{ }^{-1} \mathrm{LDL}$, top and $0.132 \mathrm{mg} \mathrm{mL}^{-1} \mathrm{HDL}$, bottom) to $2 \mathrm{~mol} \%$ DM5PS SLBs in three contrasts, dTris (green), CMTris (orange), and hTris (blue). Data and fits from the original bilayers are underlaid in pale colours. For clarity, data for CMTris and dTris are offset by 10 and 1000 , respectively. 


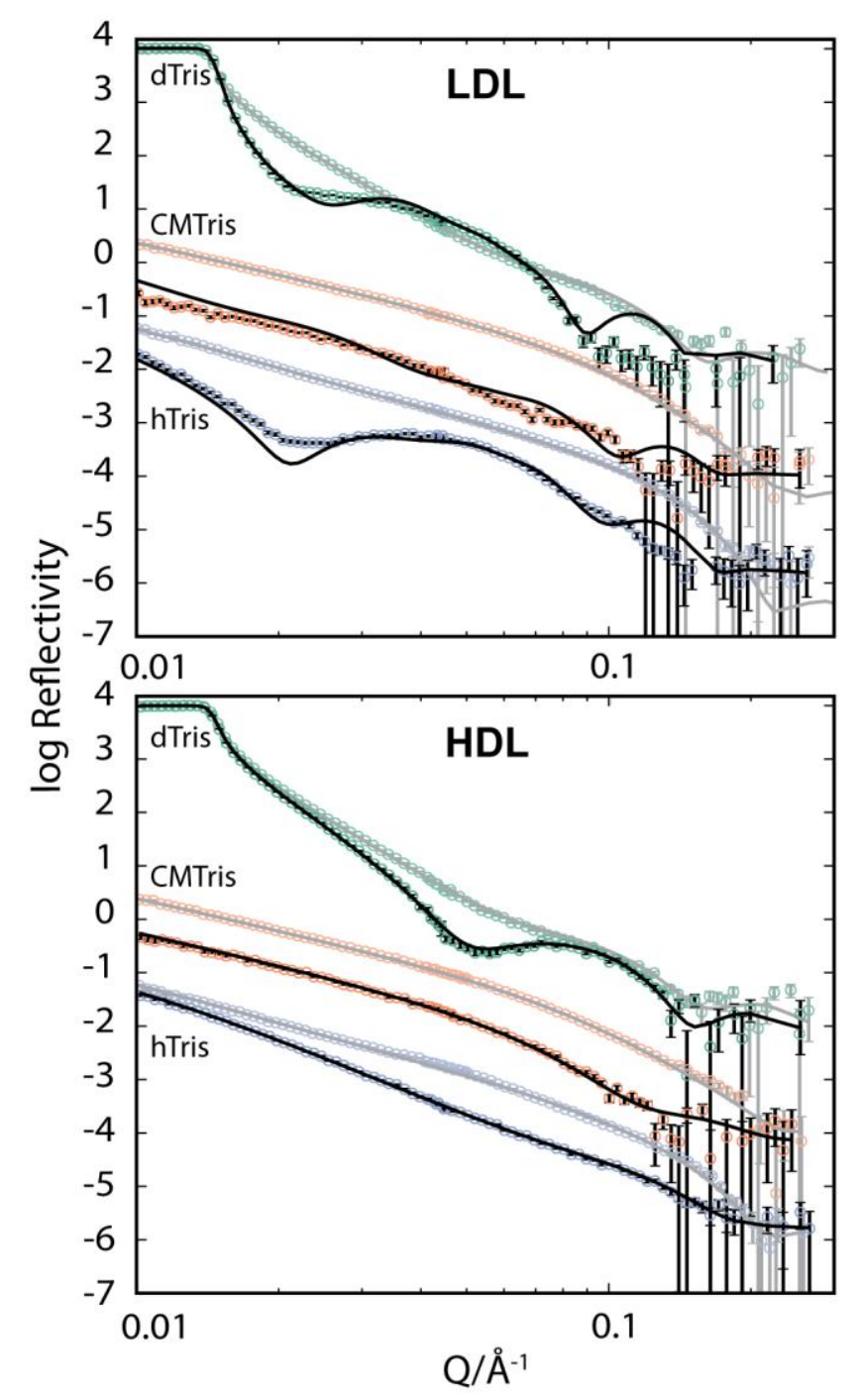

Figure 3 (dark markers and black lines) illustrates the effect on the reflectivity of incubation of 25PS SLBs with either LDL (top graph) or HDL (bottom graph). The change in reflectivity for the SLB incubated with LDL was different when compared with the other data sets, especially in hTris contrast (Figure 3 top, blue markers) where a clear fringe minimum can be seen at $0.02 \mathrm{~A}^{-1}$. In contrast, a featureless drop is seen for HDL as well as for the other, less charged bilayers (0 mol\% DMPS, $10 \mathrm{~mol} \%$ DMPS). A small drop in reflectivity at $0.02 \mathrm{~A}^{-1}$ compared to $0.05 \mathrm{~A}^{-1}$ was detected in the hTris contrast in the first measurement after injection $(<5$ minutes), suggesting that the lipoprotein-induced destruction of the SLB structure is extremely fast (Supplementary Information, Figure SI2). This is in agreement with the QCM-D data on the same system (Figure 1). Indeed, the minimum in the reflectivity 
was already evident after 3 hours (Supplementary Information, Figure SI3). The magnitude of structural change is illustrated by the fact that the data obtained from LDL incubation could not be fitted using the same model as described above, or any model preserving the head-tail-head bilayer structure. The best model was found using a 6-layer model and allowing all parameters to vary within rational limits including SLD, which clearly does not allow for detailed structural quantification. However, from the fitted SLD profile (Supplementary Information, Figure 1) and the position of the Kiessig fringe, it can be deduced that the centre of hydrogenous mass of the adsorbed material lies away from the surface at around $100 \AA$. This corresponds quite well with the observed radius of LDL particles. Thus, the LDL particles seem to remain adsorbed to the surface of the silicon crystal after complete destruction of the lipid bilayer. These results are in line with the high affinity for silica surfaces displayed by LDL.[9, 34]

The reflectivity data for 25 mol\% DMPS bilayers incubated with HDL (Figure 3, bottom) was similar to that of 0 mol\% DMPS and 10 mol\% DMPS. Thus, HDL is considerably less destructive to the DMPC/DMPS SLB than LDL. The outer head group layer was found to be larger and less hydrated than for 0 mol\% DMPS or 10 mol\% DMPS which, coupled with a thinner adsorbed layer model, suggests some interpenetration and flattening of the lipoprotein into the lipid head groups induced by the negative charge. The lower dissipation seen in the QCM-D data for 25 mol\% DMPS is in agreement with this result, as lower dissipation would be expected for a more compact adsorbed structure.

The SLD of the tail region is found to be $4.45 \times 10^{-6} \AA^{-1}$, corresponding to $30 \% \pm 1 \%$ exchange of deuterated lipids from the bilayer with hydrogenous material from HDL. The reproducibility of the calculated lipid exchange suggests that the exchange, i.e., the replacement of deuterated lipids in the lipid bilayers with hydrogenous material from the 
lipoproteins into the bilayer tail region, depends only weakly on bilayer charge. In contrast, lipoprotein-induced removal was found to be $54 \%$, which is considerably higher than that observed for HDL incubated with 0 mol\% DMPS bilayers (40\%) but very similar to the result obtained for $10 \mathrm{~mol} \%$ DMPS bilayers (52\%). Thus, addition of negative charge to the bilayer initially increases HDL-induced removal of deuterated lipids from the SLB, an effect saturating at $10 \mathrm{~mol} \%$ of DMPS in the bilayer.

Figure 4 summarises the removal of deuterated lipids (darker colours) and deposition of hydrogenous material (i.e., replacement of bilayer lipids with hydrogenous material from LDL and HDL; lighter colours) for all experiments. Lipid removal was calculated by combining the percentage of lipids replaced by hydrogenous material and solvent, giving the total amount of deuterated molceules removed from the SLB during the experiment. It is clear that the amount of material deposited into the bilayer depends only weakly on bilayer charge. This result is especially interesting considering that, although the particle concentration is kept constant between the LDL and HDL samples, due to the difference in size and composition LDL particles contain twice the number of lipids compared to HDL.[9] In contrast, the number of lipids removed from the bilayer does seem to have a clear link to bilayer charge. Removal by both HDL and LDL increases when moving from zwitterionic to negatively charged bilayers with lipid removal always being higher for HDL. In the case of LDL, however, when $25 \mathrm{~mol} \%$ DMPS is present the lipid removal is enough to destroy the entire bilayer structure.

Figure 4: Change in the bilayer tail region after incubation of dDMPC bilayers with increasing amounts of dDMPS. Red bars indicate the change after incubation with HDL and blue bars after LDL. Darker colours show the percentage of deuterated lipids removed from the bilayer and paler colours show the amount of hydrogenous material deposited into the bilayer tail region (calculated from the fitted tail SLD). The difference between removal and deposition is the percentage of 
water calculated to be in the tail region. *No data is shown for $25 \mathrm{~mol} \%$ DMPS with LDL as the bilayer structure was destroyed after incubation.

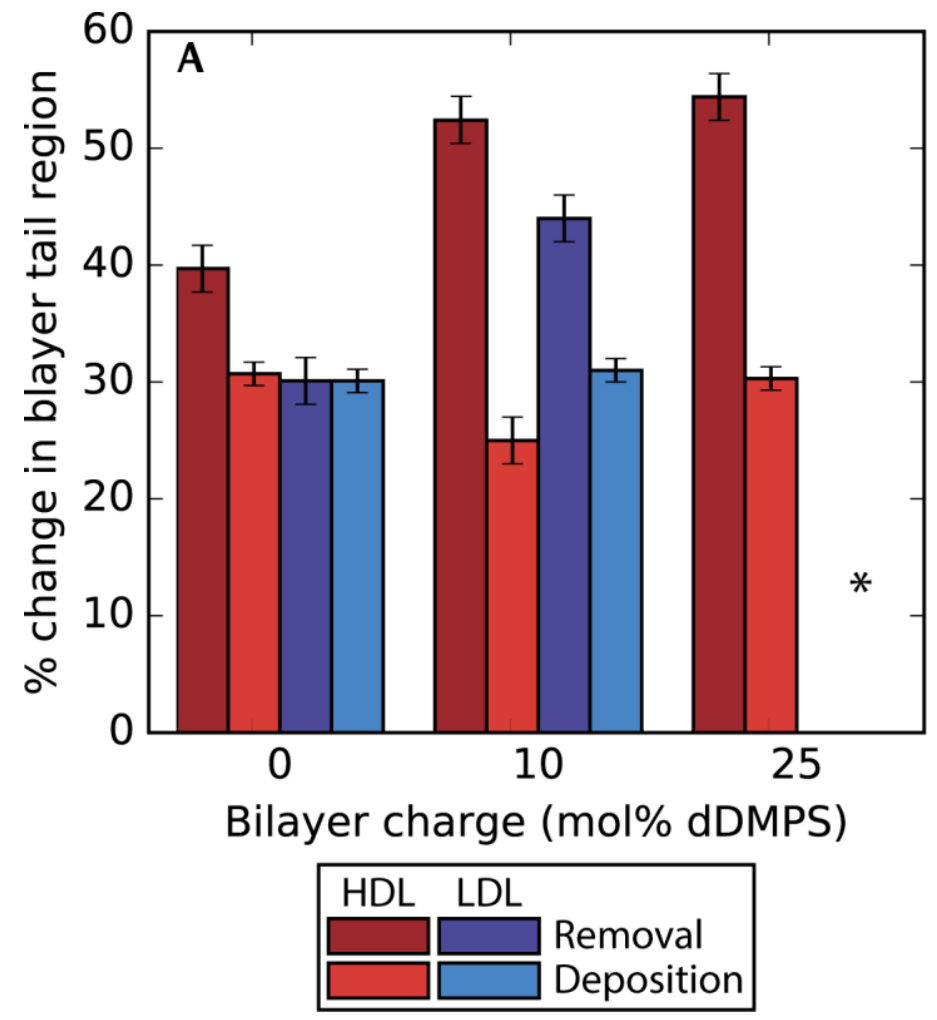

Finally, Figure 5 shows the kinetics of deuterated lipid removal as calculated from the fitted hTris contrast data recorded during incubation (Supplementary Information, Figure SI3). Overall, for both LDL and HDL, the kinetics of lipoprotein-induced removal of deuterated lipids from the SLB was found to increase with increasing charge density of the latter.

Figure 5: Time resolved removal of deuterated lipids from dDMPC bilayers of different compositions, 0 mol\% DMPS (squares), $10 \mathrm{~mol} \%$ DMPS (circles)[9] and $25 \mathrm{~mol} \%$ DMPS (triangles). The data points are calculated from hTris measurements over 8 hours of lipoprotein incubation. Data from bilayers incubated with HDL is shown in red and LDL in blue. The grey box indicates the point of washing where the lipoproteins are removed from solution and exchange slows down. *No data is shown for 25 mol\% DMPS with LDL as the bilayer structure was destroyed after incubation. 


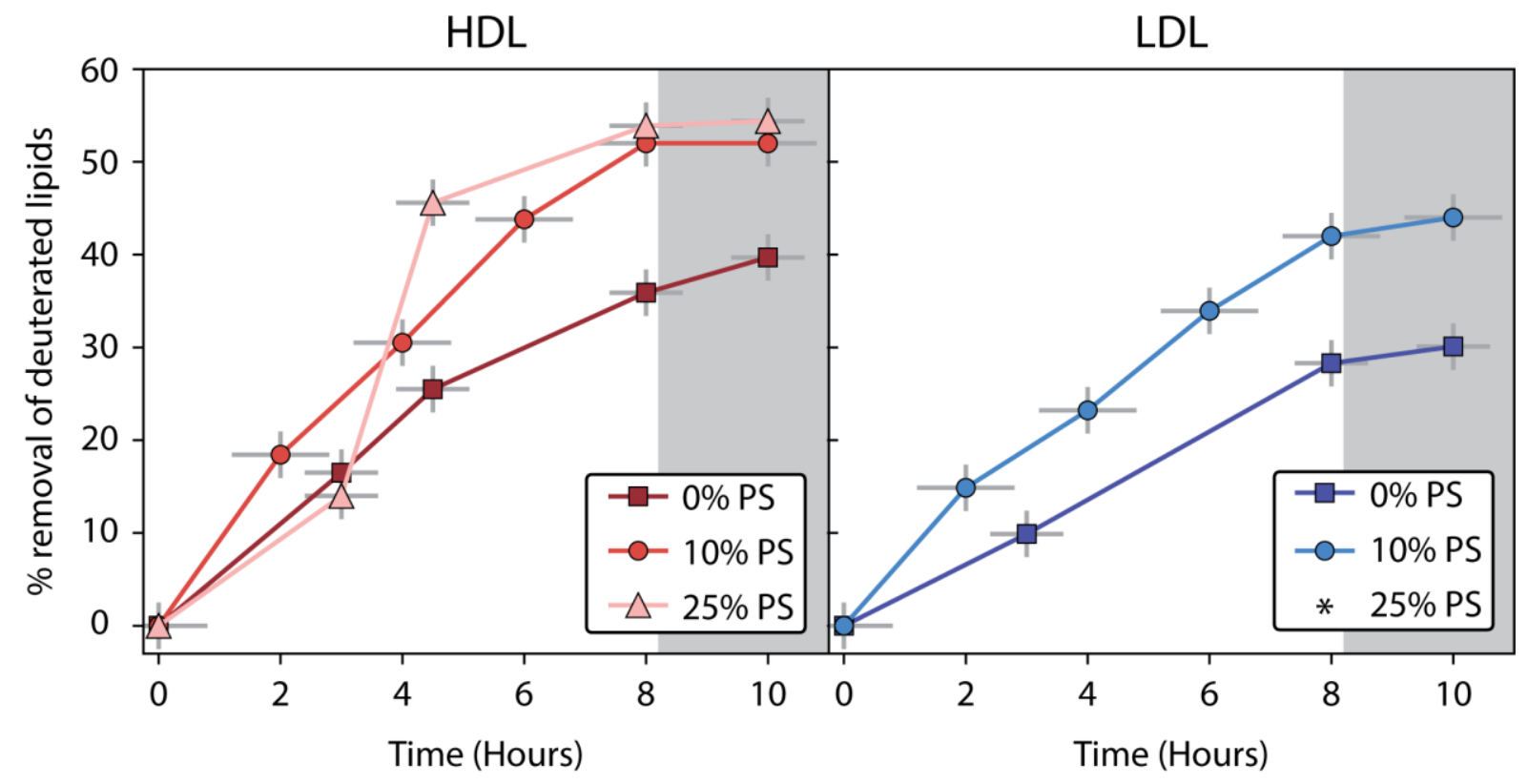

\section{Discussion}

Due to the importance of lipoprotein binding to endothelial cell membranes as the initial step of atherosclerosis, $[2,35]$ adsorption of both lipoproteins and apolipoproteins have attracted interest in previous studies in literature. In relation to the effects of electrostatics on lipoprotein-surface interactions, considerable interest has been devoted to lipoprotein binding to negatively charged materials, mediated by cationic domains within apolipoproteins.[36] Such extensive binding of lipoproteins to negatively charged surfaces has been successfully employed to remove lipoproteins through blood filtration (apheresis) using polyanionic macromolecules, such as heparin, dextran sulfate, and sulfated poly(vinyl alcohol)).[14, 16, 17] From the successful use of a wide range of polyanionic polyelectrolytes for lipoprotein binding in apheresis, it is clear that increased negative surface charge accelerates lipoprotein binding. Similarly, in studies of lipoprotein adsorption to model solid surfaces, Wang et al. found that sodium alginate sulfate surface modification of polysulfone ultrafiltration membranes displayed LDL adsorption largely reflecting their z-potential. Furthermore, absorbed LDL could be (partially) desorbed at higher ionic strength, demonstrating the 
importance of electrostatic interactions for LDL binding.[37] As demonstrated by Fang et al.[38] and by Li et al.[39], however, not only electrostatics affect LDL binding to surfaces, but also the nature of the anionic groups since glycosylation coupled with sulfonation was found to adsorb more LDL than either separately. Analogously, Malmsten et al. found apolipoprotein B, the key protein component in LDL, to display much higher binding to bilayers formed by anionic phosphatidic acid membranes than to zwitterionic phosphatidylcholine ones (z-potential -35 and $-8 \mathrm{mV}$, respectively), but also that different anionic phospholipid head groups bind LDL to different extents.[40] In line with this, both the initial adsorption kinetics and the extent of LDL binding to supported DMPC/DMPS bilayers was found to increase with increasing content of anionic DMPS in the present investigation. Interestingly, however, increasing DMPS content resulted in a maximum in the QCM-D dissipation response at 10 mol\% DMPS, indicating that membrane charge density affects also the (transient) interfacial structure of the composite membrane. This, in turn, may affect direct lipid exchange, but also apolipoprotein conformation,[41] previously demonstrated to play a key role for lipid exchange.[42] Together, these effects may contribute to the increase in the fraction of membrane lipids removed/displaced by lipoproteins observed in terms of difference in membrane charge.

Both lipid exchange and net transfer between lipoproteins of different types have been demonstrated to occur for phospholipids, cholesterol, and sphingomyelin.[43-46] Although apolipoproteins play an important role in such processes, lipid exchange has been reported to be not strictly dependent on protein exchange.[47-50] In such cases, the role of the protein seems to be primarily one of promoting lipoprotein anchoring and membrane fusion, rather than carrying the lipids from the lipoproteins to the bilayer.[51] Having said that, it should be noted that previous studies have focused primarily on exchange between lipoprotein particles, while much less is known on the exchange between lipoproteins and lipid 
monolayers/bilayers. In a rare exception from this, Laggner et al. employed neutron scattering to investigate LDL deuterated in the phospholipid head group region through exchange with phosphatidylcholine-N(CD3)3-apolipoprotein A complexes.[52] For this system, lipid exchange was associated with a net transfer of phosphatidylcholine to LDL. Furthermore, essentially all endogenous phosphatidylcholine, including lysophosphatidylcholine, and about one-third of the sphingomyelin was found to be exchangeable.[52] The same authors also employed neutron scattering with microemulsion model systems to study LDL interactions. They focused on structural arrangement of cholesteryl esters in LDL, the latter labeled by in vitro exchange with two different deuterated cholesteryl esters, one labeled in the fatty acyl chain (cholesteryl myristate-d27) and the other in the branched side chain of cholesterol (cholesteryl-25,26,27-d7 oleate).[53] Furthermore, exchange between LDL and vesicles was demonstrated by Jackson et al., using bovine liver phosphatidylcholine exchange protein to transfer $\mathrm{di}\left[{ }^{14} \mathrm{C}\right]$ palmitoylphosphatidylcholine from sonicated vesicles to human plasma LDL,[54] while Illingworth demonstrated similar lipoprotein-induced lipid exchange for human prostatic epithelial cells.[55] In neither of those previous investigations were effects of membrane charge density addressed, however. Given this, the present findings of pronounced effects of membrane charge density on lipoprotein-induced lipid removal, but weak dependence on replacement fill a gap in the literature on lipoprotein-membrane interactions.

Zhu et. al. employed QCM-D to study of the effect of charge on the exchange between SLBs and vesicles of opposite charge by tracking electrostatic adsorption of the vesicles followed by desorption as the charged lipids reached equilibrium. They found that increasing the charge density of the SLB resulted in an increased rate of lipid exchange and initial adsorption of vesicles to the SLBs. This is in good agreement with our results even though 
the study by Zhu et al did not include lipoproteins.[56] This furthermore suggests that electrostatics determine the extent of lipoprotein binding to the SLB surface.

Finally, it is important to note, that effects of lipid charge in atherosclerotic plaque formation are quite complex. For example, long-term hyperlipidaemia and type 2 diabetic patients have been reported to display a reduction in anionic groups in endothelial cells in atherosclerotic lesion-prone regions, possibly correlating increased lipid removal with increasing lipid charge density (observed in the present study) with increased occurrence of atherosclerotic lesions[18-20, 46, 47]. However, increased lipid charge density can be achieved also by lipid oxidation, constituting a risk factor for atherosclerosis.[2, 35] As a result of the latter, antioxidant treatment has been demonstrated to result in reduced atherosclerotic plaque formation in clinical trials.[57] Hence, the effects observed on lesions may be affected by composition-dependent inflammation rather than by direct lipid exchange effects. For a more complete understanding of the effects of membrane charge density on plaque formation, studies comparing different types of lipid charge, as well as concomitant changes in acyl group properties (e.g., oxidation-triggered modifications of the membrane acyl groups) are needed, as are studies of membranes containing also proteoglycans to allow for competitive lipoprotein binding to the different membrane compartments. Having said this, the present study nevertheless demonstrates that membrane charge does affect lipid removal of lipid membranes as well as the gross conformation of lipoproteins at such membranes, and that neutron reflectometry together with QCM-D offers opportunities to decipher the complex processes of simultaneous adsorption, lipid deposition (from lipoprotein to membrane) and removal (from membrane to lipoprotein). 


\section{Conclusions}

The effect of bilayer charge on the adsorption of lipoprotein, as well as lipid removal and deposition, was studied for DMPC/DMPS bilayers of varying content of anionic DMPS using neutron reflection and quartz crystal microbalance with dissipation. As such, the study extends previous findings on the role of LDL and HDL in lipid removal from, and exchange with, membrane lipids,[9] through addressing the effects of membrane charge on these processes. From neutron reflection studies at multiple contrasts, lipoprotein-induced removal of deuterated lipids from the bilayer could be differentiated from exchange of deuterated lipids with hydrogenous material from the lipoproteins. In all bilayers, the deposition of hydrogenous material, from the lipoproteins to the bilayer, was found to be largely independent of membrane charge density or lipoprotein class. In contrast, lipid removal in the presence of HDL was lower in zwitterionic bilayers, suggesting that the lipid uptake mechanism of HDL was impeded at reduced membrane charge, as indeed found in atherosclerotic plaque prone regions of long-term hyperlipidaemia and type 2 diabetic patients.[18-20] While simplistic, these findings thus seem relevant also for more complex atherosclerotic plaque formation.

\section{Acknowledgements}

We wish to thank the Institut Laue-Langevin (experiment no. 9-13-609, doi:10.5291/ILLDATA.9-13-609, data available Dec 2018) and ISIS neutron source (experiment no. RB1610149, doi:10.5286/isis.e.73941712) for the allocation of experimental beam time. We also wish to thank Gunilla Fredrikson and Eva Bengtsson for valuable discussion and input in setting up the project and Ann-Margreth Carlsson and Ingrid Söderberg for isolation of the 
lipoproteins. This work was supported by the Swedish Research Council (project grant numbers 2014-3981 (KB, TKL, SM, MC) and 2016-05157 (MM), and the LEO Foundation Centre for Cutaneous Drug Delivery (2016-11-01) (KB, MM). The authors declare no conflicts of interest.

\section{References}

1. WHO, Global Health Estimates 2015: Deaths by Cause, Age, Sex, by Country and by Region, 2000-2015. 2016, World Health Organization: Geneva.

2. Ross, R., Atherosclerosis--an inflammatory disease. N Engl J Med, 1999. 340(2): p. 115-26.

3. Mendis, S., et al., Global Atlas on cardiovascular disease prevention and control, WHO, Editor. 2011, World Health Organization: Geneva.

4. Prassl, R. and P. Laggner, Molecular structure of low density lipoprotein: current status and future challenges. Eur Biophys J, 2009. 38(2): p. 145-58.

5. Fogelstrand, P. and J. Boren, Retention of atherogenic lipoproteins in the artery wall and its role in atherogenesis. Nutr Metab Cardiovasc Dis, 2012. 22(1): p. 1-7.

6. Navab, M., et al., HDL and cardiovascular disease: atherogenic and atheroprotective mechanisms. Nat Rev Cardiol, 2011. 8(4): p. 222-32.

7. Fragneto, G. and M. Rheinstädter, Structural and dynamical studies from bio-mimetic systems: an overview. Comptes Rendus Physique, 2007. 8(7-8): p. 865-883.

8. Ruggeri, F., et al., Non-specific interactions between soluble and induce irreversible changes in the properties of bilayers. Soft Matter, 2013. 9(16): p. 4219-26.

9. Browning, K.L., et al., Human Lipoproteins at Model Cell Membranes: Effect of Lipoprotein Class on Lipid Exchange. Scientific Reports, 2017. 7(1): p. 7478.

10. Pasquale, L., et al., An experimental test of new theoretical models for the electrokinetic properties of biological membranes. The effect of $\mathrm{UO}_{2++}$ and tetracaine on the electrophoretic mobility of bilayer membranes and human erythrocytes. J Gen Physiol, 1986. 88(6): p. 697-718.

11. Ribeiro, M.M.B., et al., Chemical Conjugation of the Neuropeptide Kyotorphin and Ibuprofen Enhances Brain Targeting and Analgesia. Molecular Pharmaceutics, 2011. 8(5): p. 19291940.

12. Bosch, T., et al., $L D L$ hemoperfusion--a new procedure for $L D L$ apheresis: first clinical application of an LDL adsorber compatible with human whole blood. Artif Organs, 1997. 21(9): p. 977-82.

13. Assogba, U., et al., Blood antioxidants (vitamin E and beta-carotene) in long-term low density lipoprotein apheresis. Clin Chim Acta, 1995. 235(2): p. 147-57.

14. Maaskant, N., A. Bantjes, and H.J.M. Kempen, Removal of low density lipoprotein from blood plasma using cross-linked, sulfated polyvinylalcohol. Atherosclerosis, 1986. 62(2): p. 159166.

15. Nakajima, T., et al., A novel system of $L D L$ apheresis combining a centrifugal plasma separator with a specific LDL adsorption column. Atherosclerosis, 1988. 73(2-3): p. 143-148.

16. Ando, S. and M. Matsuzaki, Isolation of Low Density Lipoprotein Subfraction Containing Apolipoprotein B-like Protein from Japanese Eel Anguilla japonica Plasma Using Dextran Sulfate Cellulose. Comparative Biochemistry and Physiology Part B: Biochemistry and Molecular Biology, 1997. 116(2): p. 191-196. 
17. Thompson, G.R., LDL apheresis. Atherosclerosis, 2003. 167(1): p. 1-13.

18. Ghinea, N., et al., Prelesional events in atherogenesis. Changes induced by hypercholesterolemia in the cell surface chemistry of arterial endothelium and blood monocytes, in rabbit. J Submicrosc Cytol, 1987. 19(2): p. 209-27.

19. Manduteanu, I. and M. Simionescu, Inflammation in atherosclerosis: a cause or a result of vascular disorders? J Cell Mol Med, 2012. 16(9): p. 1978-90.

20. Nassimizadeh, M., et al., Reduced negative surface charge on arterial endothelium explains accelerated atherosclerosis in type 2 diabetic patients. Diab Vasc Dis Res, 2010. 7(3): p. 2135.

21. Hui, S.W., et al., Identification of Phosphatidylserine and Phosphatidy/choline in CalciumInduced Phase Separated Domains. Biochemistry, 1983. 22(14): p. 3511-3516.

22. Bradford, M.M., A rapid and sensitive method for the quantitation of microgram quantities of protein utilizing the principle of protein-dye binding. Anal Biochem, 1976. 72: p. 248-54.

23. Garrett, R.H. and C.M. Grisham, Lipid Biosynthesis, in Biochemistry. 2016, Cengage Learning: Boston, USA. p. 862.

24. Jagalski, V., et al., Biophysical study of resin acid effects on phospholipid membrane structure and properties. Biochim Biophys Acta, 2016. 1858(11): p. 2827-2838.

25. Cho, N.J., et al., Quartz crystal microbalance with dissipation monitoring of supported lipid bilayers on various substrates. Nat Protoc, 2010. 5(6): p. 1096-106.

26. Campbell, R.A., et al., FIGARO: The new horizontal neutron reflectometer at the ILL. The European Physical Journal Plus, 2011. 126(11).

27. Campbell, R.A., et al., Erratum to: FIGARO: The new horizontal neutron reflectometer at the ILL. The European Physical Journal Plus, 2015. 130(10).

28. Nelson, A., Motofit-integrating neutron reflectometry acquisition, reduction and analysis into one, easy to use, package. Journal of Physics: Conference Series, 2010. 251.

29. Tellechea, E., et al., Model-independent analysis of QCM data on colloidal particle adsorption. Langmuir, 2009. 25(9): p. 5177-84.

30. Nagle, J.F. and S. Tristram-Nagle, Structure of lipid bilayers. Biochimica et Biophysica Acta (BBA) - Reviews on Biomembranes, 2000. 1469(3): p. 159-195.

31. Hellstrand, E., et al., Adsorption of alpha-synuclein to supported lipid bilayers: positioning and role of electrostatics. ACS Chem Neurosci, 2013. 4(10): p. 1339-51.

32. Melcrova, A., et al., The complex nature of calcium cation interactions with phospholipid bilayers. Sci Rep, 2016. 6: p. 38035.

33. Petrache, H.I., et al., Structure and Fluctuations of Charged Phosphatidylserine Bilayers in the Absence of Salt. Biophysical Journal, 2004. 86(3): p. 1574-1586.

34. Malmsten, M., G. Siegel, and W.G. Wood, Ellipsometry Studies of Lipoprotein Adsorption. Journal of Colloid and Interface Science, 2000. 224(2): p. 338-346.

35. Tabas, I., G. Garcia-Cardena, and G.K. Owens, Recent insights into the cellular biology of atherosclerosis. J Cell Biol, 2015. 209(1): p. 13-22.

36. Hazelrig, J.B., M.K. Jones, and J.P. Segrest, A mathematically defined motif for the radial distribution of charged residues on apolipoprotein amphipathic alpha helixes. Biophysical Journal, 1993. 64(6): p. 1827-1832.

37. Wang, W., et al., Immobilization of sodium alginate sulfates on polysulfone ultrafiltration membranes for selective adsorption of low-density lipoprotein. Acta Biomaterialia, 2014. 10(1): p. 234-243.

38. Fang, F., et al., Anionic glycosylated polysulfone membranes for the affinity adsorption of low-density lipoprotein via click reactions. Acta Biomaterialia, 2017. 49: p. 379-387.

39. Li, J., et al., Bioinspired Multiple-Interaction Model Revealed in Adsorption of Low-Density Lipoprotein to Surface Containing Saccharide and Alkanesulfonate. Langmuir, 2013. 29(26): p. 8363-8369. 
40. Malmsten, M., et al., Adsorption of Apolipoprotein B at Phospholipid Model Surfaces. Journal of Colloid and Interface Science, 1995. 172(2): p. 485-493.

41. Raghavendra, A.J., et al., Charge-transfer interactions induce surface dependent conformational changes in apolipoprotein biocorona. Biointerphases, 2017. 12(2): p. $02 \mathrm{D} 402$.

42. Córsico, B., J.D. Toledo, and H.A. Garda, Evidence for a Central Apolipoprotein A-I Domain Loosely Bound to Lipids in Discoidal Lipoproteins That Is Capable of Penetrating the Bilayer of Phospholipid Vesicles. Journal of Biological Chemistry, 2001. 276(20): p. 16978-16985.

43. Illingworth, D.R. and O.W. Portman, Independence of Phospholipid and Protein Exchange between Plasma Lipoproteins in-Vivo and in-Vitro. Biochimica Et Biophysica Acta, 1972. 280(2): p. 281-+.

44. Kunkel, H.G. and A.G. Bearn, Phospholipid Studies of Different Serum Lipoproteins Employing P32. Proceedings of the Society for Experimental Biology and Medicine, 1954. 86(4): p. 887891.

45. Eder, H.A., The lipoproteins of human serum. The American Journal of Medicine, 1957. 23(2): p. 269-282.

46. Illingworth, D.R. and O.W. Portman, Exchange of Phospholipids between Low and HighDensity Lipoproteins of Squirrel-Monkeys. Journal of Lipid Research, 1972. 13(2): p. 220-+.

47. Bilheimer, D.W., S. Eisenberg, and R.I. Levy, The metabolism of very low density lipoprotein proteins I. Preliminary in vitro and in vivo observations. Biochimica et Biophysica Acta (BBA) Lipids and Lipid Metabolism, 1972. 260(2): p. 212-221.

48. Eisenberg, S. and D. Rachmilewitz, Metabolism of rat plasma very low density lipoprotein. Biochimica et Biophysica Acta (BBA) - Lipids and Lipid Metabolism, 1973. 326(3): p. 378-390.

49. Eisenberg, S. and D. Rachmilewitz, Metabolism of rat plasma very low density lipoprotein. Biochimica et Biophysica Acta (BBA) - Lipids and Lipid Metabolism, 1973. 326(3): p. 391-405.

50. Eisenberg, S., D.W. Bilheimer, and R.I. Levy, The metabolism of very low density lipoprotein proteins. Biochimica et Biophysica Acta (BBA) - Lipids and Lipid Metabolism, 1972. 280(1): p. 94-104.

51. Khattari, Z., Adsorption kinetics of low-density lipoproteins with Langmuir monolayer. J Biol Phys, 2016. 42(4): p. 539-550.

52. Laggner, P., et al., Neutron small angle scattering on selectively deuterated human plasma low density lipoproteins. The location of polar phospholipid headgroups. J Biol Chem, 1981. 256(22): p. 11832-9.

53. Laggner, P., et al., Structure of the cholesteryl ester core of human plasma low density lipoproteins: selective deuteration and neutron small-angle scattering. Proc Natl Acad Sci U S A, 1984. 81(14): p. 4389-93.

54. Jackson, R.L., et al., Incorporation of dipalmtoyl phosphatidy/choline into human plasma low density lipoproteins. Biochimica et Biophysica Acta (BBA) - Lipids and Lipid Metabolism, 1980. 619(2): p. 408-413.

55. Illingworth, D.R., et al., The exchange of phospholipids between plasma lipoproteins and rapidly dividing human cells grown in tissue culture. Biochimica et Biophysica Acta (BBA) Lipids and Lipid Metabolism, 1973. 306(3): p. 422-436.

56. Zhu, T., Z. Jiang, and Y. Ma, Lipid exchange between membranes: Effects of membrane surface charge, composition, and curvature. Colloids and Surfaces B: Biointerfaces, 2012. 97: p. 155-161.

57. Rodriguez, M., et al., Reduction of atherosclerotic nanoplaque formation and size by Ginkgo biloba (EGb 761) in cardiovascular high-risk patients. Atherosclerosis, 2007. 192(2): p. 43844. 


\section{Highlights}

\section{Effect of bilayer charge on lipoprotein lipid exchange}

- Charge effects on lipoprotein-induced changes in membrane structure and composition

- Neutron reflection able to distinguish between lipid exchange and removal

- Deposition from lipoprotein is not dependant on lipoprotein type or bilayer charge

- Lipid removal increases with bilayer charge for both LDL and HDL

- Correlates with findings on atherosclerotic plaque in type 2 diabetic patients 